\title{
Probability of Soluble Tissue Factor Release Lead to the Elevation of D-dimer as a Biomarker for Traumatic Brain Injury
}

\author{
Eiichi SuEHIRO, ${ }^{1}$ Yuichi FuJIYAMA, ${ }^{1}$ Miwa KIYOHIRA, ${ }^{1}$ Yukari MOTOKI, ${ }^{2}$ \\ Junzo NOJIMA, ${ }^{2}$ and Michiyasu SUZUKI ${ }^{1}$ \\ ${ }^{1}$ Department of Neurosurgery, Yamaguchi University Graduate School of Medicine, \\ Ube, Yamaguchi, Japan; \\ ${ }^{2}$ Department of Laboratory Science, Faculty of Health Science, Yamaguchi \\ University Graduate School of Medicine, Ube, Yamaguchi, Japan
}

\begin{abstract}
D-dimer is a potential biomarker for the detection of traumatic brain injury (TBI). However, the mechanisms that trigger elevation of D-dimer in TBI remain unclear. The purpose of this study was to evaluate the reliability of D-dimer in blood as a biomarker for TBI and to determine the mechanisms involved in regulating its blood levels. Nine patients with moderate to severe isolated TBI (Glasgow Coma Scale [GCS] score 7-13) were admitted to our hospital from May 2013 to June 2014. Blood samples were collected from systemic arteries on arrival and at 1, 3, 5, and 7 days after injury. Blood levels of neuron specific enolase (NSE), D-dimer, and soluble tissue factor (sTF) were measured. NSE (33.4 ng/ml: normal $<12.0 \mathrm{ng} / \mathrm{ml}$ ) and D-dimer (56.1 $\mu \mathrm{g} / \mathrm{ml}$ : normal $<1.0 \mu \mathrm{g} / \mathrm{ml})$ were elevated at admission and declined on day 1 after injury. At admission, there were significant correlations of D-dimer levels with NSE $(R=0.727, P=0.026)$ and sTF $(R=0.803, P=0.009)$ levels. The blood level of D-dimer accurately reflects the degree of brain tissue damage indicated by NSE levels. Our data suggest that release of STF induced by brain tissue damage may activate the coagulation cascade, leading to elevation of D-dimer.
\end{abstract}

Key words: biomarker, D-dimer, neuron specific enolase, tissue factor, traumatic brain injury

\section{Introduction}

Outcomes in patients with trauma have been improved by development of pre-hospital care and initial treatment, ${ }^{1)}$ but outcomes of traumatic brain injury (TBI) still remain poor. There has been little progress on treatment of severe TBI, which makes it important to prevent progression of mild or moderate TBI to severe disease. This approach requires prediction of the risk of progression of TBI, and this might be achieved using serum biomarkers such as glial fibrillary acidic protein (GFAP), S100B protein and UCH-L1 (ubiquitin carboxyl-terminal hydrolase-L1). ${ }^{2)}$ These biomarkers can be used to indicate lesions in the skull, predict outcomes in TBI, and assist with determination of therapeutic strategies. ${ }^{3-6)}$

Received October 23, 2018; Accepted December 10, 2018

Copyright $\odot 2019$ by The Japan Neurosurgical Society This work is licensed under a Creative Commons AttributionNonCommercial-NoDerivatives International License.
We have reported prediction of outcomes in patients with isolated TBI using plasma D-dimer as a biomarker. ${ }^{7,8}$ Plasma D-dimer has the advantage of being an inexpensive and convenient test item that is already used in clinical practice; however, it has a weakness of being affected by injuries other than intracranial injury and coagulation disorder. Elevation of D-dimer is caused by TBI-induced abnormalities in the coagulation and fibrinolytic system; however, the mechanism remains unclear. ${ }^{9,10)}$ Hypotheses of the mechanism of coagulation disorder after trauma include dilution of blood due to major bleeding and infusion, resulting in a shortage of coagulation factor; ${ }^{11,12)}$ hypotension-induced protein $C$ activation and fibrinolysis activation; ${ }^{13)}$ and an extrinsic coagulation cascade that is activated by tissue factors released from damaged brain tissues due to TBI, leading to induction of fibrinolysis. ${ }^{14)}$ In a previous study, we found that patients with mild $\mathrm{TBI}^{8)}$ are unlikely to have major bleeding, infusion or hypotension. In this study, we examined TBI with a focus on tissue factors. 
The above hypotheses were proposed before it was shown that tissue factors are involved in elevation of plasma D-dimer in TBI. ${ }^{15,16)}$ Coagulation cascade activation followed by release of tissue factors as an indirect consequence have been assumed to occur based on the low fibrinogen level with increased levels of fibrin(ogen)-related antigen, and the low levels of factors $\mathrm{V}$ and VIII and platelets found in patients with TBI. ${ }^{15,16)}$ However, there are few studies of direct measurement of soluble tissue factor (sTF) in serum of patients with TBI. Therefore, we measured sTF in patients with TBI and examined its relationship with plasma D-dimer. Neuron specific enolase (NSE) was simultaneously measured as a TBI biomarker, and the relationship between severity of brain injury and plasma D-dimer levels was examined. The results allow evaluation of the utility of the plasma D-dimer level for prediction of intracranial lesions in patients with mild $\mathrm{TBI}^{8}$ ) and for evaluation of disease progression during follow-up. ${ }^{7)}$

\section{Patients and Methods}

A retrospective observational cohort study was performed in nine patients (all male) who were admitted with moderate to severe isolated TBI and a Glasgow Coma Scale (GCS) score at admission of 7-13 from May 2013 to June 2014. All patients were treated in the Department of Neurosurgery based on Japan Society of Neurotraumatology guidelines ${ }^{17)}$ for management of severe TBI. The patients had a mean age of 51.7 years, with a range of 6-87 years. The protocol was approved by the Institutional Review Board of Yamaguchi University School of Medicine, which is a level I trauma center. All patients gave informed consent to participate in the study.

If needed, patients underwent craniotomy for evacuation of hematoma, and decompressive craniectomy to control intracranial pressure. Peripheral arterial blood samples were collected at admission and on days $1,3,5$, and 7 after admission in a tube containing EDTA (ethylenediaminetetraacetic acid) for separation of serum or containing sodium citrate $(3.2 \%, 0.11 \mathrm{~mol} / \mathrm{L})$ for separation of plasma. Samples were centrifuged immediately after collection (10 min, $3000 \mathrm{rpm}$ ) at $4^{\circ} \mathrm{C}$, and supernatants were collected as serum and plasma. The concentration of D-dimer in plasma was measured with a latex immunoassay (LIA) kit (LIAS AUTO D-dimer NEO, Sysmex, Hyogo, Japan), as routinely performed in our hospital. Serum samples were stored at $-85^{\circ} \mathrm{C}$ until analysis. NSE in serum as an indicator of brain tissue damage was measured with an electrochemiluminescence immunoassay for human NSE kit (Roche Diagnostics K.K., Tokyo, Japan), and sTF in serum was determined using an enzymelinked immunosorbent assay for human tissue factor (Assaypro LLC, St. Charles, MO, USA).

Correlations of D-dimer levels with NSE and sTF levels were evaluated by Pearson linear regression to evaluate $\mathrm{D}$-dimer as a biomarker for brain tissue damage and to determine if release of STF is associated with D-dimer elevation, respectively. Correlations were considered as significant at $P<0.05$.

\section{Results}

Individual data for the nine patients in the study, including age, gender, type of injury, GCS score on admission, and Glasgow Outcome Scale (GOS) score at discharge are shown in Table 1. NSE levels in serum (Fig. 1) greatly exceeded the normal range

Table 1 Summary of patients

\begin{tabular}{lccccc}
\hline Case & Age (years) & Gender & Diagnosis & GCS & GOS \\
\hline 1 & 6 & Male & AEDH & 12 & GR \\
2 & 87 & Male & ASDH, CC & 7 & SD \\
3 & 33 & Male & ASDH & 11 & MD \\
4 & 72 & Male & AEDH, CC & 13 & MD \\
5 & 62 & Male & ASDH, CC & 7 & MD \\
6 & 44 & Male & CC, t-SAH & 9 & GR \\
7 & 42 & Male & AEDH & 13 & GR \\
8 & 51 & Male & AEDH & 11 & GR \\
9 & 68 & Male & ASDH & 13 & GR \\
\hline
\end{tabular}

GCS: Glasgow Coma Scale score on admission, GOS: Glasgow Outcome Scale at discharge, GR: good recovery, MD: moderate disability, SD: severe disability, AEDH: acute epidural hematoma, ASDH: acute subdural hematoma, CC: cerebral contusion, t-SAH: traumatic subarachnoid hemorrhage.

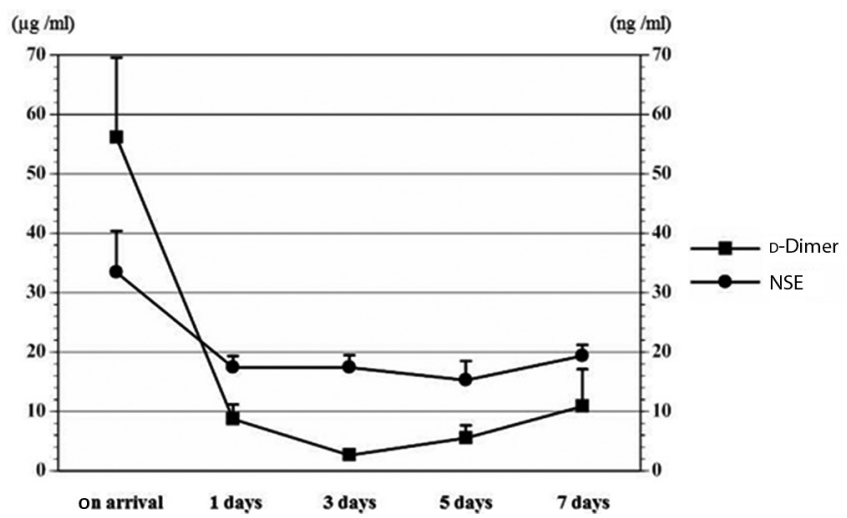

Fig. 1 Changes in NSE and D-dimer concentrations in serum or plasma over the study period. The concentration of NSE and D-dimer was extremely high at admission, but decreased on the next day. Values are mean \pm SEM. NSE: neuron specific enolase. 
$(<12.0 \mathrm{ng} / \mathrm{ml})$ at admission, but this increase was completely attenuated on the following day. Similarly, D-dimer in plasma (Fig. 1) exceeded the normal range $(<1.0 \mu \mathrm{g} / \mathrm{ml})$ at admission, but then decreased markedly. At admission, D-dimer levels were significantly correlated with NSE levels $(R=0.727, P=0.026$; Fig. 2) and with blood levels of $\operatorname{sTF}(R=0.803$, $P=0.009$; Fig. 3).

\section{Discussion}

In this study, blood D-dimer levels at admission were evaluated to determine their utility as a biomarker for severity of TBI. These levels were correlated with those of NSE, which is an established biomarker for TBI. This suggests that D-dimer is an effective biomarker for TBI. Blood D-dimer levels were also significantly correlated with those of sTF. Based on these results, sTF released from damaged brain tissues is probably involved in elevation of D-dimer in isolated TBI. This is the first study to

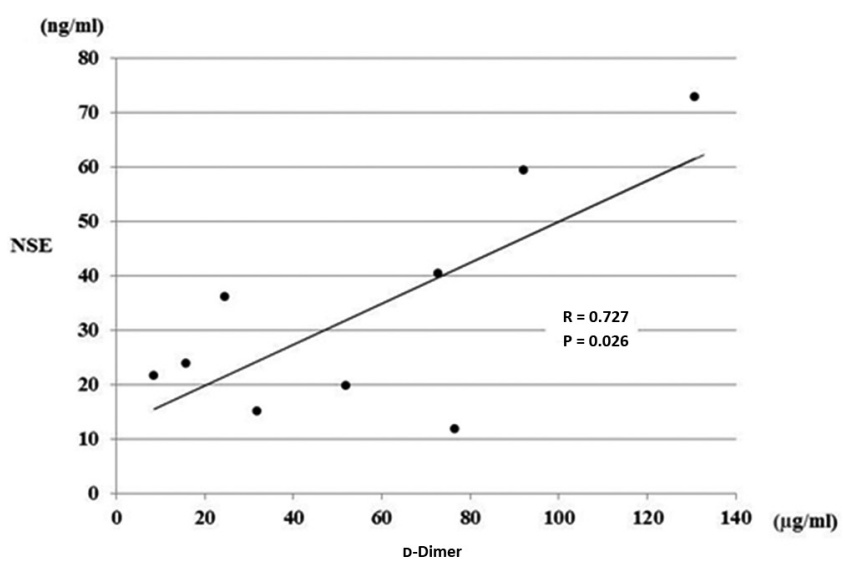

Fig. 2 Linear regression curve of NSE and D-dimer levels in blood at admission. NSE: neuron specific enolase.

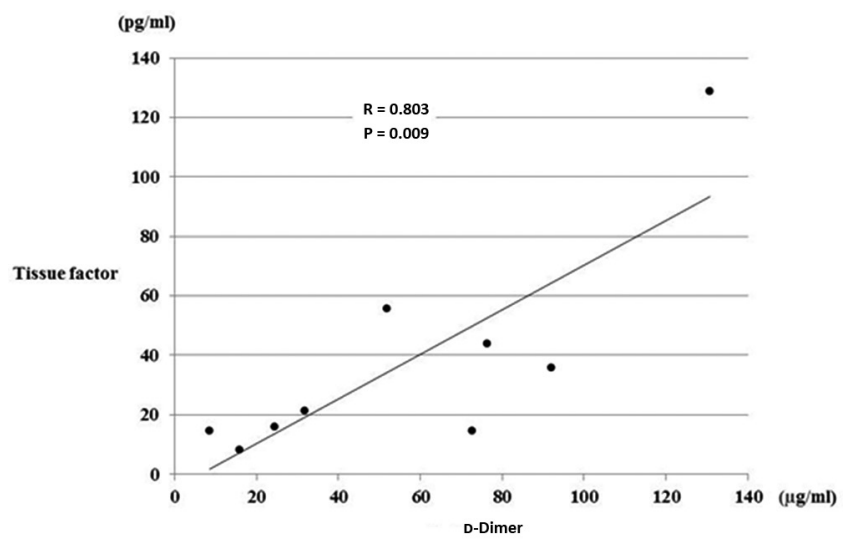

Fig. 3 Linear regression curve of D-dimer and soluble tissue factor levels in blood at admission. show a relationship between D-dimer and sTF in TBI patients. The mechanism underlying this relationship is still unclear, but the association of sTF with elevation of D-dimer lends credibility to use of D-dimer as a biomarker for TBI. These results are important because the D-dimer test is inexpensive and routinely performed in clinical practice, which will facilitate its introduction for patients with TBI.

Neuron specific enolase is commonly used as a tumor marker, and is also an enzyme in the cytoplasm of neurons. Therefore, an increased plasma NSE level can indicate release into the blood due to neuronal damage, ${ }^{2)}$ and high NSE is related to poor outcomes of TBI. ${ }^{2,3,5,6)}$ However, the accuracy of NSE as a biomarker for neural injury is inferior to that of other biomarkers, such as S100B, GFAP and UCH-L1.6) In this study, plasma NSE was strongly elevated at admission, but then markedly lower on the next day, after which it remained at around the upper limit of the normal range for several more days. Intracranial hypertension and reduced brain perfusion pressure during the course of TBI sometimes cause secondary brain injury, and serum S100B and NSE may increase again during treatment, reflecting this secondary injury. ${ }^{4)}$ The stability of NSE levels during treatment in this study suggests that intracranial pressure was controlled well and secondary brain injury was minimized, with the result of good outcomes for eight out of nine subjects. There was no significant correlation between NSE and GCS score on admission or GOS at discharge. Less brain parenchyma damage, especially for acute epidural hematoma, induces difficulty to understanding of correlation between a value of biomarker and the level of consciousness. Brain dysfunction due to compression of the hematoma or complication of the axonal injury may induce to the elevation of the value of biomarker. Further, this may be because eight subjects had good recovery (GR) and evaluation with GOS was difficult. Different results might have been obtained in evaluation of higher brain function. Also, the level of consciousness and functional prognosis depend on localization of brain damage, while a biomarker quantifies brain injury, which makes it difficult to compare GOS and biomarkers. It seems to be the need for the accumulation of the number of cases.

Hemorrhage control is important in treatment of systemic trauma, but is often difficult due to coagulopathy after trauma. ${ }^{18)}$ Prediction of the severity and outcomes of trauma using coagulation markers is based on this phenomenon. The injury severity score of trauma patients is correlated with fibrin degradation product (FDP) and D-dimer on arrival, ${ }^{19)}$ and the severity of coagulation and fibrinolytic system disorder 
after trauma is related to the severity of trauma, ${ }^{20)}$ as also found in the current study. These findings raise the question of whether it is possible to predict the severity and outcomes of TBI using coagulation markers. There are three major hypotheses for the mechanism of coagulopathy after trauma: that massive transfusion and infusion in initial treatment after trauma dilute the blood, resulting in a shortage of blood coagulation factors; ${ }^{11,12)}$ that hypotension induces protein $\mathrm{C}$ activation, which activates fibrinolysis; ${ }^{13)}$ and that tissue factors are released from TBI-damaged brain tissues into the blood, and the coagulation cascade is then activated by these factors. ${ }^{14)}$ We have shown a relationship of brain injury with D-dimer levels in mild isolated TBI in previous studies, ${ }^{7,8)}$ and patients without blood dilution due to massive transfusion and infusion or hypotension during treatment still have abnormalities in the coagulation and fibrinolytic system $^{21)}$ that are not associated with the mechanism of coagulation disorder after TBI. The results of this study suggest that STF is involved in the mechanism of coagulopathy after TBI. Release of tissue factors from a damaged brain into blood causes a thrombin burst, and this thrombin binds to thrombomodulin to produce a thrombin-thrombomodulin complex and activate protein C. Activated protein C then inhibits plasminogen activator inhibitor-1 and induces hyperfibrinolysis.

Traumatic brain injury patients have abnormal PT, APTT (activated partial thromboplastin time), FDP and D-dimer levels, and these coagulation markers are predictors of outcomes. ${ }^{22-25)}$ However, the underlying mechanisms are unknown. Based on decreased fibrinogen and platelets and increased thrombin-antithrombin III complex (TAT) and prothrombin fragment F1 +2 in patients with TBI, it has been suggested that activation of an "extrinsic" pathway with sTF as a trigger is involved in TBI. ${ }^{15,16)}$ Therefore, it is significant that involvement of sTF in elevation of D-dimer was shown in this study, and this supports use of D-dimer as a TBI biomarker. D-dimer is routinely measured in clinical practice in Japan, whereas use of other biomarkers, including S-100B and GFAP, is uncommon due to the difficulty and expense of measurements. However, D-dimer has a disadvantage of low specificity because it is also increased by injuries and thrombosis at other sites. Thus, D-dimer may be useful only in the acute phase of isolated TBI.

\section{Conclusion}

The results of this study suggest that STF is associated with elevation of D-dimer in TBI. This direct relationship of the increase in $\mathrm{D}$-dimer to brain injury provides evidence for use of $\mathrm{D}$-dimer as a TBI biomarker. D-dimer measurement is simple and inexpensive in clinical practice, and may be useful in determining a therapeutic strategy for TBI. However, D-dimer may also increase in patients with multiple injuries and thrombosis, and this may limit its use as a biomarker to patients with acute isolated TBI.

\section{Acknowledgments}

This work was supported by research funds from JSPS KAKENHI Grant Number 16K10725 and ZENKYOREN (National Mutual Insurance Federation of Agricultural Cooperatives).

\section{Conflicts of Interest Disclosure}

All authors have no conflicts of interest.

\section{References}

1) Meizoso JP, Valle EJ, Allen CJ, et al.: Decreased mortality after prehospital interventions in severely injured trauma patients. J Trauma Acute Care Surg 79: 227-231, 2015

2) Lorente L: Biomarkers associated with the outcome of traumatic brain injury patients. Brain Sci 7: E142, 2017

3) Cheng F, Yuan Q, Yang J, Wang W, Liu H: The prognostic value of serum neuron-specific enolase in traumatic brain injury: systematic review and meta-analysis. PLOS ONE 9: e106680, 2014

4) Olivecrona Z, Bobinski L, Koskinen LO: Association of ICP, CPP, CT findings and S-100B and NSE in severe traumatic head injury. Prognostic value of the biomarkers. Brain Inj 29: 446-454, 2015

5) Rodríguez-Rodríguez A, Egea-Guerrero JJ, GordilloEscobar E, et al.: S100B and Neuron-Specific Enolase as mortality predictors in patients with severe traumatic brain injury. Neurol Res 38: 130-137, 2016

6) Thelin EP, Jeppsson E, Frostell A, et al.: Utility of neuron-specific enolase in traumatic brain injury; relations to S100B levels, outcome, and extracranial injury severity. Crit Care 20: 285, 2016

7) Suehiro E, Koizumi H, Fujiyama Y, Yoneda H, Suzuki M: Predictors of deterioration indicating a requirement for surgery in mild to moderate traumatic brain injury. Clin Neurol Neurosurg 127: 97-100, 2014

8) Sugimoto K, Suehiro E, Shinoyama M, et al.: d-dimer elevation as a blood biomarker for detection of structural disorder in mild traumatic brain injury. J Neurotrauma 34: 3245-3248, 2017

9) Bredbacka S, Edner G: Soluble fibrin and d-dimer as detectors of hypercoagulability in patients with isolated brain trauma. J Neurosurg Anesthesiol 6: 75-82, 1994 
10) Scherer RU, Spangenberg P: Procoagulant activity in patients with isolated severe head trauma. Crit Care Med 26: 149-156, 1998

11) Geeraedts LM, Demiral H, Schaap NP, Kamphuisen PW, Pompe JC, Frölke JP: 'Blind' transfusion of blood products in exsanguinating trauma patients. Resuscitation 73: 382-388, 2007

12) Holcomb JB, Jenkins $D$, Rhee $P$, et al.: Damage control resuscitation: directly addressing the early coagulopathy of trauma. J Trauma 62: 307-310, 2007

13) Brohi K, Cohen MJ, Ganter MT, Matthay MA, Mackersie RC, Pittet JF: Acute traumatic coagulopathy: initiated by hypoperfusion: modulated through the protein C pathway? Ann Surg 245: 812-818, 2007

14) Zhang J, Jiang R, Liu L, Watkins T, Zhang F, Dong JF: Traumatic brain injury-associated coagulopathy. J Neurotrauma 29: 2597-2605, 2012

15) Keimowitz RM, Annis BL: Disseminated intravascular coagulation associated with massive brain injury. J Neurosurg 39: 178-180, 1973

16) Goodnight SH, Kenoyer G, Rapaport SI, Patch MJ, Lee JA, Kurze T: Defibrination after brain-tissue destruction: a serious complication of head injury. $N$ Engl J Med 290: 1043-1047, 1974

17) Shigemori M, Abe T, Aruga T, et al.; Guidelines Committee on the Management of Severe Head Injury, Japan Society of Neurotraumatology: Guidelines for the Management of Severe Head Injury, 2nd Edition guidelines from the Guidelines Committee on the Management of Severe Head Injury, the Japan Society of Neurotraumatology. Neurol Med Chir (Tokyo) 52: 1-30, 2012

18) Kutcher ME, Ferguson AR, Cohen MJ: A principal component analysis of coagulation after trauma. J Trauma Acute Care Surg 74: 1223-1229; discussion 1229-1230, 2013

19) Hagiwara S, Oshima K, Aoki M, et al.: Usefulness of fibrin degradation products and d-dimer levels as biomarkers that reflect the severity of trauma. J Trauma Acute Care Surg 74: 1275-1278, 2013

20) Hayakawa M, Maekawa K, Kushimoto S, et al.: High d-dimer levels predict a poor outcome in patients with severe trauma, even with high fibrinogen levels on arrival: a multicenter retrospective study. Shock 45: 308-314, 2016

21) Hayakawa M, Maekawa K, Kushimoto S, et al.: Hyperfibrinolysis in severe isolated traumatic brain injury may occur without tissue hypoperfusion: a retrospective observational multicentre study. Crit Care 21: 222, 2017

22) Kuo JR, Chou TJ, Chio CC: Coagulopathy as a parameter to predict the outcome in head injury patients-analysis of 61 cases. J Clin Neurosci 11: 710-714, 2004

23) Genét GF, Johansson PI, Meyer MA, et al.: Traumainduced coagulopathy: standard coagulation tests, biomarkers of coagulopathy, and endothelial damage in patients with traumatic brain injury. J Neurotrauma 30: 301-306, 2013

24) Chhabra G, Sharma S, Subramanian A, Agrawal D, Sinha S, Mukhopadhyay AK: Coagulopathy as prognostic marker in acute traumatic brain injury. J Emerg Trauma Shock 6: 180-185, 2013

25) DeFazio MV, Rammo RA, Robles JR, Bramlett HM, Dietrich WD, Bullock MR: The potential utility of blood-derived biochemical markers as indicators of early clinical trends following severe traumatic brain injury. World Neurosurg 81: 151-158, 2014

Address reprint requests to: Eiichi Suehiro, MD, PhD, Department of Neurosurgery, Yamaguchi University School of Medicine, 1-1-1 Minamikogishi, Ube, Yamaguchi 755-8505, Japan. e-mail: suehiro-nsu@umin.ac.jp 